\title{
ONE PARAMETER FAMILY OF LINEAR DIFFERENCE EQUATIONS AND THE STABILITY PROBLEM FOR THE NUMERICAL SOLUTION OF ODES
}

\author{
L. ACETO, R. PANDOLFI, AND D. TRIGIANTE
}

Received 21 July 2004; Accepted 4 October 2004

The study of the stability properties of numerical methods leads to considering linear difference equations depending on a complex parameter $q$. Essentially, the associated characteristic polynomial must have constant type for $q \in \mathbb{C}^{-}$. Usually such request is proved with the help of computers. In this paper, by using the fact that the associated polynomials are solutions of a "Legendre-type" difference equation, a complete analysis is carried out for the class of linear multistep methods having the highest possible order.

Copyright (c) 2006 Hindawi Publishing Corporation. All rights reserved.

\section{Introduction}

The problem to approximate the solutions of differential equations by substituting to them "appropriate" difference equations is as old as the differential calculus. Of course the main problem to be solved is the control of the errors between the continuous and the discrete solutions. In the fifties, the fundamental importance of the stability properties of the difference equations on the error propagation was recognized. After that, a lot of efforts has been done in this field, mainly when the methods are applied to dissipative problems. In such a case, the first approximation theorem permits to transform the nonlinear problem into a linear one. Regarding the class of linear multistep methods (LMMs), the propagation of the errors can be studied by means of a linear difference equation which, in the scalar case, depends on a complex parameter $q=h \lambda$, where $h$ is the stepsize and $\lambda$ is the derivative at the critical point of the function defining the differential equation. Obviously, the characteristic polynomial of the derived difference equation also depends on the same parameter $q$.

The order of the equation of the error is, usually, greater than the one of the differential equation. Therefore, an higher number of conditions are needed to get the solution we are interested in. When all the conditions are fixed at the beginning of the interval of integration, it is well-known that the asymptotic stability of the zero solution of the error equation is equivalent to require that the characteristic polynomial is a Schur polynomial, that is all its roots lie in the unit circle, for all $q \in \mathbb{C}^{-}$. On the contrary, when the conditions are split between the beginning and the end of the interval of integration, the concept of 
stability needs to be generalized. In such a case the notion of well-conditioning is more appropriate. Essentially, such notion requires that under the perturbation $\delta \eta$ of the imposed conditions, the perturbation of the solution $\delta \mathrm{y}$ should be bounded as follows:

$$
\|\delta \mathbf{y}\| \leq \mathscr{K}\|\delta \eta\|,
$$

where $\mathscr{Y}$ is independent on the number of points in the discrete mesh.

It is worth to note that when the discrete equation is of higher order with respect to the continuous initial value problem, it is not necessary to approximate the latter with a discrete problem of the same type. In the case of first order differential equation, for example, only the condition $y_{0}$ is provided by the continuous problem. The additional conditions are at our disposal and we may fix them at our convenience. This is important because the well-known Dahlquist barriers impose several limitations on the choice of the methods in the class of LMMs used as initial value methods (IVMs). As matter of fact, there are not methods with order greater than two having the critical point asymptotically stable for all $q \in \mathbb{C}^{-}$. When a discrete linear boundary value problem is considered, the error equation is well-conditioned if the number of initial conditions is equal to the number of roots of the characteristic polynomial inside the unit circle and the number of conditions at the end of the interval of integration is equal to the number of roots outside the unit circle [2]. Once again, we will have a well-conditioned problem when a fixed set of roots remains constantly inside the unit circle for all $q \in \mathbb{C}^{-}$. This result generalizes the stability condition for IVMs where the roots inside need to be all of them.

In order to control that the number of roots inside the unit circle is constant for $q \in$ $\mathbb{C}^{-}$, special importance assume the unit circumference and its image in the complex $q$ plane (said boundary locus) under an appropriate map defined by each method. Except for very simple cases, the proof that the number of roots inside the unit circle remains constant for all $q \in \mathbb{C}^{-}$is only made by using the boundary locus pictures, provided by computers.

In this paper we will consider the family of highest order methods in the class of LMMs and we will give a complete proof that they are well-conditioned when used as boundary value methods (BVMs), while, as proved in [3], they are unstable when used as IVMs. The analysis will be done by studying a special linear difference equation with variable coefficients (essentially the one satisfied by Legendre polynomials).

The paper is structured as follows: in Section 2 we will generalize the classical stability concept for IVMs and we will introduce the highest possible order methods in the class of LMMs. In Section 3 some properties of the polynomials associated to these methods will be analyzed and, in Section 4, the related stability problem will be discussed. Finally, some conclusions will be stated in Section 5 .

\section{The problem of stability for boundary value methods}

As mentioned in the Introduction, the study of the stability for dissipative nonlinear problems is made by linearizing the nonlinearity around the asymptotically stable critical point. This is equivalent to examine the behavior of the solutions of a numerical method 
when applied to the test equation

$$
y^{\prime}(t)=\lambda y(t), \quad t \in\left[t_{0}, T\right], \quad \operatorname{Re} \lambda<0,
$$

subject to the initial condition $y\left(t_{0}\right)=y_{0}$.

By using a consistent $k$-step linear multistep method (LMM) on the discrete set $\left\{t_{\ell}\right\}$ defined by

$$
t_{\ell}=t_{0}+\ell h, \quad \ell=0,1, \ldots, N, h=\frac{T-t_{0}}{N}
$$

we get the following difference equation of order $k$ :

$$
\sum_{j=0}^{k}\left(\alpha_{j}-q \beta_{j}\right) y_{n+j}=0, \quad q=h \lambda, \quad k \geq 1,
$$

where $y_{n+j}, n=0,1, \ldots, N-k$, approximates the value $y\left(t_{n+j}\right)$ of the continuous solution at the grid point $t_{n+j}$.

Denoting by $e_{n}=y\left(t_{n}\right)-y_{n}$ the error at $t_{n}$, from the previous relation we obtain the unperturbed error equation:

$$
\sum_{j=0}^{k}\left(\alpha_{j}-q \beta_{j}\right) e_{n+j}=0, \quad q=h \lambda .
$$

In order to study the stability of the zero solution of this equation, we consider the characteristic polynomial defined by

$$
\pi(z, q)=\rho(z)-q \sigma(z)
$$

where

$$
\rho(z)=\sum_{j=0}^{k} \alpha_{j} z^{j}, \quad \sigma(z)=\sum_{j=0}^{k} \beta_{j} z^{j} .
$$

It is well-known that when (2.4) is coupled with $k$ initial conditions, the asymptotic stability of its zero solution is equivalent to require that the characteristic polynomial $\pi(z, q)$ is a Schur polynomial for all $q \in \mathbb{C}^{-}$. Such a choice of the additional conditions leads to very severe restrictions on the order of methods (Dahlquist barriers). To overcome this drawback, since only $y_{0}$ is provided by the continuous problem, we can choose to fix some of the $(k-1)$ additional conditions at the beginning of the interval of integration and the remaining at the end. Later on, we will refer to the discrete problem obtained by associating to (2.3) $k_{1}$ initial conditions and $k_{2}=k-k_{1}$ final ones as boundary value method (BVM) with $\left(k_{1}, k_{2}\right)$-boundary conditions [2].

This allows to extend the classical stability concept. To this aim we consider the definition of type of a polynomial due to Miller [6]. 
Definition 2.1. A polynomial of degree $k$ is said to be of type $\left(r_{1}, r_{2}, r_{3}\right)$ if it has $r_{1}$ zeros inside the unit circle, $r_{2}$ zeros with unit modulus and $r_{3}$ zeros outside the unit circle, with $r_{1}, r_{2}, r_{3}$ and $k$ non-negative integers such that $k=r_{1}+r_{2}+r_{3}$.

The following definition is the generalization to the case $k_{2}>0$ of the corresponding well-known concepts valid only for the case $k_{2}=0$.

Definition 2.2. A BVM with $\left(k_{1}, k_{2}\right)$-boundary conditions is said to be

- $A_{k_{1}, k_{2}}$ stable if $\mathbb{C}^{-} \subseteq \mathscr{D}_{k_{1} k_{2}}$, where

$$
\mathscr{D}_{k_{1} k_{2}}=\left\{q \in \mathbb{C}: \pi(z, q) \text { is of type }\left(k_{1}, 0, k_{2}\right)\right\}
$$

denotes the region of $\left(k_{1}, k_{2}\right)$-Absolute stability;

- Perfectly $A_{k_{1}, k_{2}}$-stable if $\mathbb{C}^{-} \equiv \mathscr{D}_{k_{1} k_{2}}$.

Remark 2.3. The terminology used in Numerical Analysis is often different from the one used in the Difference Equations setting. In order to avoid confusion, it is worth to note that the terms such as "stable methods" refer to the well-conditioning of the error equation and not necessarily to the stability of the zero solution of the same equation. It is known that the well-conditioning of a linear boundary value problem, either continuous or discrete, is related to the so called dichotomy. In the discrete case it essentially states that the number of initial conditions should be equal to the number of roots of the characteristic polynomial inside the unit circle and, of course, the number of conditions at the end of the interval of integration should be equal to the number of roots outside the unit circle.

Since our equations depend on the parameter $q$, the dichotomy should remain constant for all $q$ of interest, that is, $q \in \mathbb{C}^{-}$. Considering that the type of the polynomial varies only if a root crosses the unit circle, an efficient way to establish if a numerical method verifies the stability concepts given in Definition 2.2 is the knowledge of the form and the position in the complex plane of the set

$$
\Gamma=\left\{q \in \mathbb{C}: \pi\left(e^{i \theta}, q\right)=0, \theta \in[0,2 \pi)\right\},
$$

called boundary locus. The equation $\pi(z, q)=0$ defines a map between the complex $z$ plane and the complex $q$-plane, that is,

$$
q(z)=\frac{\rho(z)}{\sigma(z)}
$$

consequently,

$$
\Gamma=\left\{q \in \mathbb{C}: q=q\left(e^{i \theta}\right), \theta \in[0,2 \pi)\right\} .
$$

The request that the type of $\pi(z, q)$ should not change for $q \in \mathbb{C}^{-}$is equivalent to $\Gamma \cap \mathbb{C}^{-}=$ $\varnothing$. Usually, unless for simple cases, $\Gamma$ is obtained graphically. In the sequel, we will give an analytic expression of the boundary locus associated to each $k$-step LMM having highest possible order, that is $2 k$, and constituting the so called Top Order family. These methods 
are characterized by the following polynomials of degree $k$ (see (2.6))

$$
\rho_{k}(z)=\sum_{j=0}^{k} \alpha_{j}^{(k)} z^{j}, \quad \sigma_{k}(z)=\sum_{j=0}^{k} \beta_{j}^{(k)} z^{j}
$$

having real coefficients given by

$$
\alpha_{j}^{(k)}=2\left[\sum_{r=1}^{j} \frac{1}{r}-\sum_{r=1}^{k-j} \frac{1}{r}\right] \beta_{j}^{(k)}, \quad \beta_{j}^{(k)}=\frac{[k !]^{2}}{(2 k) !}\left(\begin{array}{l}
k \\
j
\end{array}\right)^{2}, \quad j=0,1, \ldots, k .
$$

Essentially, these coefficients were obtained by Dahlquist in [3] (in this more explicit form they can be found in [1]). It is an easy matter to prove that they satisfy the following relations of symmetry:

$$
\alpha_{j}^{(k)}=-\alpha_{k-j}^{(k)}, \quad \beta_{j}^{(k)}=\beta_{k-j}^{(k)}, \quad j=0,1, \ldots, k .
$$

Preliminary properties concerning $\rho_{k}(z)$ and $\sigma_{k}(z)$ can be now established.

Lemma 2.4. The polynomials $\rho_{k}(z)$ and $\sigma_{k}(z)$ with coefficients given by (2.12) satisfy the following properties:

(1)

$$
\rho_{k}(z)=-z^{k} \rho_{k}\left(z^{-1}\right), \quad \sigma_{k}(z)=z^{k} \sigma_{k}\left(z^{-1}\right) .
$$

(2) $\rho_{k}(1)=0$ for all $k \geq 1, \rho_{k}(-1)=0$ for $k$ even, $\sigma_{k}(-1)=0$ for $k$ odd.

(3) If $z_{j}$ is any other root, different from 1 and -1 , of each of them, so is $z_{j}^{-1}$.

(4) The function

$$
\hat{\rho}_{k-1}(z)=\frac{\rho_{k}(z)}{z-1}
$$

is a symmetric polynomial, that is,

$$
\hat{\rho}_{k-1}(z)=z^{k-1} \hat{\rho}_{k-1}\left(z^{-1}\right),
$$

having positive coefficients.

Proof. Item (1) immediately follows by taking into account (2.11) and by using (2.13). Moreover, (2) and (3) are easy consequence of (2.14). Concerning item (4), from (2.14) we obtain

$$
\hat{\rho}_{k-1}(z)=\frac{-z^{k} \rho_{k}\left(z^{-1}\right)}{z-1}=\frac{z^{k-1} \rho_{k}\left(z^{-1}\right)}{z^{-1}-1}=z^{k-1} \hat{\rho}_{k-1}\left(z^{-1}\right) .
$$

Since $\hat{\rho}_{k-1}(z)$ is a symmetric polynomial, in order to prove that its coefficients are all positive, it is sufficient to evaluate the sign of the first $v$ of them, where

$$
v= \begin{cases}\frac{k}{2} & \text { for even } k, \\ \frac{k+1}{2} & \text { for odd } k .\end{cases}
$$


6 Difference equations and the stability problem

By posing

$$
\hat{\rho}_{k-1}(z)=\sum_{j=0}^{k-1} a_{j}^{(k-1)} z^{j}
$$

from (2.15) we get

$$
\sum_{j=1}^{k} a_{j-1}^{(k-1)} z^{j}-\sum_{j=0}^{k-1} a_{j}^{(k-1)} z^{j}=\sum_{j=0}^{k} \alpha_{j}^{(k)} z^{j}
$$

This implies that

$$
\begin{gathered}
a_{j}^{(k-1)}=a_{j-1}^{(k-1)}-\alpha_{j}^{(k)}, \quad j=1, \ldots, \nu-1, \\
a_{0}^{(k-1)}=-\alpha_{0}^{(k)} .
\end{gathered}
$$

Considering that $\alpha_{0}^{(k)}$ and $\alpha_{j}^{(k)}$ are negative quantities (see (2.12)), the previous relations give the thesis.

From the above results we have that $\sigma_{k}(z)$ and $\hat{\rho}_{k-1}(z)$ have many basic common properties. Some of them can be summarized as follows:

symmetry: $u_{\kappa}(z)=z^{\kappa} u_{\kappa}\left(z^{-1}\right)$,

positivity: the coefficients are positive,

negative root: $u_{\kappa}(-1)=0$ for $\kappa$ odd,

where $u_{\kappa}(z)$ denotes either $\sigma_{k}(z)$ or $\hat{\rho}_{k-1}(z)$, and $\kappa$ is the degree of the involved polynomial.

Considerations based on the numerical concept of consistency permit to exclude that the roots +1 and -1 are multiple. In Section 3, however, such properties will be directly proved as consequence of the fact that $\rho_{k}(z)$ and $\sigma_{k}(z)$ satisfy a linear difference equation. For the moment we assume that the real roots of such polynomials, on the unit circle, are all simple. Concerning the map

$$
q_{k}(z)=\frac{\rho_{k}(z)}{\sigma_{k}(z)}=\frac{\hat{\rho}_{k-1}(z)(z-1)}{\sigma_{k}(z)}
$$

by taking into account the statement (3) in Lemma 2.4 we obtain

$$
q_{k}\left(e^{i \theta}\right)= \begin{cases}\left(e^{i 2 \theta}-1\right) \frac{\alpha_{k}^{(k)}}{\beta_{k}^{(k)}} \frac{\prod_{j=1}^{\nu-1}\left[e^{i 2 \theta}-\left(v_{j}+v_{j}^{-1}\right) e^{i \theta}+1\right]}{\prod_{j=1}^{v}\left[e^{i 2 \theta}-\left(w_{j}+w_{j}^{-1}\right) e^{i \theta}+1\right]} & \text { for even } k, \\ \frac{e^{i \theta}-1}{e^{i \theta}+1} \frac{\alpha_{k}^{(k)}}{\beta_{k}^{(k)}} \prod_{j=1}^{\nu-1} \frac{e^{i 2 \theta}-\left(v_{j}+v_{j}^{-1}\right) e^{i \theta}+1}{e^{i 2 \theta}-\left(w_{j}+w_{j}^{-1}\right) e^{i \theta}+1} & \text { for odd } k,\end{cases}
$$


where $v_{j}$ and $w_{j}$ are the roots of $\rho_{k}(z)$ and $\sigma_{k}(z)$, respectively, and $\nu$ is defined according to (2.18). Considering (2.12), the previous relation becomes

$$
\begin{aligned}
q_{k}\left(e^{i \theta}\right)= & \begin{cases}\left(2 \sum_{r=1}^{k} \frac{1}{r}\right)\left(e^{i \theta}-e^{-i \theta}\right) \frac{\prod_{j=1}^{v-1}\left[e^{i \theta}-\left(v_{j}+v_{j}^{-1}\right)+e^{-i \theta}\right]}{\prod_{j=1}^{v}\left[e^{i \theta}-\left(w_{j}+w_{j}^{-1}\right)+e^{-i \theta}\right]} & \text { for even } k, \\
\left(2 \sum_{r=1}^{k} \frac{1}{r}\right) \frac{e^{i(\theta / 2)}-e^{-i(\theta / 2)}}{e^{i(\theta / 2)}+e^{-i(\theta / 2)}} \prod_{j=1}^{\nu-1} \frac{e^{i \theta}-\left(v_{j}+v_{j}^{-1}\right)+e^{-i \theta}-\left(w_{j}+w_{j}^{-1}\right)+e^{-i \theta}}{e^{i \theta}} & \text { for odd } k,\end{cases} \\
= & \begin{cases}i \sin \theta\left(4 \sum_{r=1}^{k} \frac{1}{r}\right) \frac{\prod_{j=1}^{v-1}\left[2 \cos \theta-\left(v_{j}+v_{j}^{-1}\right)\right]}{\prod_{j=1}^{v}\left[2 \cos \theta-\left(w_{j}+w_{j}^{-1}\right)\right]} & \text { for even } k, \\
i \tan \frac{\theta}{2}\left(2 \sum_{r=1}^{k} \frac{1}{r}\right) \prod_{j=1}^{v-1} \frac{2 \cos \theta-\left(v_{j}+v_{j}^{-1}\right)}{2 \cos \theta-\left(w_{j}+w_{j}^{-1}\right)} & \text { for odd } k,\end{cases} \\
\doteq & \begin{array}{ll}
i \sin \theta F_{\nu}(\theta) & \text { for even } k, \\
i \tan \frac{\theta}{2} G_{\nu-1}(\theta) & \text { for odd } k .
\end{array}
\end{aligned}
$$

The following result holds true.

Theorem 2.5. Assuming that the multiplicity of the roots 1 and -1 of both polynomials $\rho_{k}(z)$ and $\sigma_{k}(z)$ is at most one, the values $F_{\nu}(\theta)$ and $G_{\nu-1}(\theta)$ are real numbers (possibly infinite) for all $\theta \in[0,2 \pi)$.

Proof. It is trivial considering that if, for example, $v_{j}$ is complex, the product will contain both term $\left(v_{j}+v_{j}^{-1}\right)$ and its conjugate. The same, of course, holds for $w_{j}$.

In order to have the type of $\pi(z, q)$ constant for all $q \in \mathbb{C}^{-}, F_{\nu}(\theta)$ and $G_{\nu-1}(\theta)$ have to preserve their sign for all $\theta \in[0,2 \pi)$. From the expressions of $F_{\nu}(\theta)$ and $G_{\nu-1}(\theta)$, it turns out that they are ratios of trigonometric polynomials. Although a large number of results involving trigonometric polynomials are known (see $[4,5,7]$, to quote only a few works related to this subject), it seems that none of them can be here applied. For this reason in the next section we will give a proof valid in our case.

\section{Further properties of the associated polynomials}

In order to state the main result, we need to establish further properties of the polynomials $\rho_{k}(z)$ and $\sigma_{k}(z)$.

In [3] Dahlquist already stressed the relation between $\sigma_{k}(z)$ defined in (2.11) (divided by a constant of normalization) and the Legendre polynomials

$$
L_{k}(x)=\left[\frac{x-1}{2}\right]^{k} \sum_{j=0}^{k}\left(\begin{array}{l}
k \\
j
\end{array}\right)^{2}\left(\frac{x+1}{x-1}\right)^{j}, \quad k=0,1,2, \ldots
$$

In fact we have the following. 
8 Difference equations and the stability problem

Lemma 3.1. Let $\sigma_{k}(z)$ be the polynomial defined in (2.11). Then, for all $k \geq 0$

$$
\sigma_{k}(z)=\frac{(k !)^{2}}{(2 k) !}(z-1)^{k} L_{k}\left(\frac{z+1}{z-1}\right) .
$$

Proof. By setting in $(3.1) z=(x+1) /(x-1)$, the thesis trivially follows by using $(2.11)$ and (2.12).

It is known that the Legendre polynomials verify the recurrence relation of the form

$$
\begin{gathered}
L_{k+1}(x)=\frac{2 k+1}{k+1} x L_{k}(x)-\frac{k}{k+1} L_{k-1}(x), \quad k \geq 1, \\
L_{0}(x)=1, \quad L_{1}(x)=x .
\end{gathered}
$$

Then, we may prove the following result.

Theorem 3.2. The polynomial $\sigma_{k}(z)$, given in (2.11), satisfies the difference equation

$$
y_{k+1}(z)=\frac{z+1}{2} y_{k}(z)-\frac{(z-1)^{2}}{4\left(4-k^{-2}\right)} y_{k-1}(z), \quad k \geq 1,
$$

with initial conditions

$$
y_{0}(z)=1, \quad y_{1}(z)=\frac{z+1}{2}
$$

Proof. From (3.2) one has

$$
\sigma_{k+1}(z)=\frac{[(k+1) !]^{2}}{(2 k+2) !}(z-1)^{k+1} L_{k+1}\left(\frac{z+1}{z-1}\right)
$$

By using (3.3) in the previous relation we get

$$
\sigma_{k+1}(z)=\frac{[(k+1) !]^{2}}{(2 k+2) !}(z-1)^{k+1}\left[\frac{2 k+1}{k+1} \frac{z+1}{z-1} L_{k}\left(\frac{z+1}{z-1}\right)-\frac{k}{k+1} L_{k-1}\left(\frac{z+1}{z-1}\right)\right] .
$$

By direct calculation it is easy to check that

$$
\frac{[(k+1) !]^{2}}{(2 k+2) !} \frac{2 k+1}{k+1}=\frac{(k !)^{2}}{2(2 k) !}, \quad \frac{[(k+1) !]^{2}}{(2 k+2) !} \frac{k}{k+1}=\frac{[(k-1) !]^{2}}{4\left(4-k^{-2}\right)(2 k-2) !} .
$$

Thus, relation (3.7) becomes

$$
\begin{aligned}
\sigma_{k+1}(z)= & \frac{z+1}{2} \frac{(k !)^{2}}{(2 k) !}(z-1)^{k} L_{k}\left(\frac{z+1}{z-1}\right)-\frac{(z-1)^{2}}{4\left(4-k^{-2}\right)} \\
& \cdot \frac{[(k-1) !]^{2}}{(2 k-2) !}(z-1)^{k-1} L_{k-1}\left(\frac{z+1}{z-1}\right) .
\end{aligned}
$$

Consequently, from Lemma 3.1 the thesis follows. 
In order to tackle the question concerning the values assumed by the polynomials $\hat{\rho}_{k-1}(z)$ and $\sigma_{k}(z)$ on the unit circle, we need to consider the following results.

Lemma 3.3. The coefficients defined in (2.12), characterizing the polynomial $\rho_{k}(z)$, satisfy the recurrence relation:

$$
\alpha_{j}^{(k+1)}=\frac{\alpha_{j-1}^{(k)}+\alpha_{j}^{(k)}}{2}-\frac{\alpha_{j-2}^{(k-1)}-2 \alpha_{j-1}^{(k-1)}+\alpha_{j}^{(k-1)}}{4\left(4-k^{-2}\right)}, \quad j=0,1, \ldots, k+1, k \geq 1,
$$

with $\alpha_{0}^{(0)}=0, \alpha_{0}^{(1)}=-1, \alpha_{1}^{(1)}=1$ and $\alpha_{s}^{(m)}=0$ for $s<0$ or $s>m$.

Proof. The proof follows by direct check.

THEOREM 3.4. The polynomial $\rho_{k}(z)$, given in (2.11), satisfies the difference equation (3.4) with initial conditions

$$
y_{0}(z)=0, \quad y_{1}(z)=z-1
$$

Proof. From the relation (3.10) we can write

$$
\sum_{j=0}^{k+1} \alpha_{j}^{(k+1)} z^{j}=\sum_{j=0}^{k+1} \frac{\alpha_{j-1}^{(k)}+\alpha_{j}^{(k)}}{2} z^{j}-\sum_{j=0}^{k+1} \frac{\alpha_{j-2}^{(k-1)}-2 \alpha_{j-1}^{(k-1)}+\alpha_{j}^{(k-1)}}{4\left(4-k^{-2}\right)} z^{j} .
$$

Considering our notational convention $\left(\alpha_{s}^{(m)}=0\right.$ whenever $s<0$ or $\left.s>m\right)$, the previous relation becomes

$$
\begin{aligned}
\sum_{j=0}^{k+1} \alpha_{j}^{(k+1)} z^{j}= & \frac{1}{2} \sum_{j=1}^{k+1} \alpha_{j-1}^{(k)} z^{j}+\frac{1}{2} \sum_{j=0}^{k} \alpha_{j}^{(k)} z^{j}-\frac{1}{4\left(4-k^{-2}\right)} \\
& \cdot\left[\sum_{j=2}^{k+1} \alpha_{j-2}^{(k-1)} z^{j}-2 \sum_{j=1}^{k} \alpha_{j-1}^{(k-1)} z^{j}+\sum_{j=0}^{k-1} \alpha_{j}^{(k-1)} z^{j}\right] \\
= & \frac{1}{2} z \sum_{j=0}^{k} \alpha_{j}^{(k)} z^{j}+\frac{1}{2} \sum_{j=0}^{k} \alpha_{j}^{(k)} z^{j}-\frac{1}{4\left(4-k^{-2}\right)} \\
& \cdot\left[z^{2} \sum_{j=0}^{k-1} \alpha_{j}^{(k-1)} z^{j}-2 z \sum_{j=0}^{k-1} \alpha_{j}^{(k-1)} z^{j}+\sum_{j=0}^{k-1} \alpha_{j}^{(k-1)} z^{j}\right] .
\end{aligned}
$$

Then, from (2.11) we deduce that

$$
\begin{aligned}
\rho_{k+1}(z) & =\frac{1}{2} z \rho_{k}(z)+\frac{1}{2} \rho_{k}(z)-\frac{1}{4\left(4-k^{-2}\right)}\left[z^{2} \rho_{k-1}(z)-2 z \rho_{k-1}(z)+\rho_{k-1}(z)\right] \\
& =\frac{z+1}{2} \rho_{k}(z)-\frac{z^{2}-2 z+1}{4\left(4-k^{-2}\right)} \rho_{k-1}(z) .
\end{aligned}
$$

This completes the proof.

Lemma 3.5. The solutions $\rho_{k}(z)$ and $\sigma_{k}(z)$ of (3.4) are linearly independent. 
10 Difference equations and the stability problem

Proof. Let us consider the Casorati matrix defined by

$$
C(k)=\left(\begin{array}{cc}
\rho_{k}(z) & \sigma_{k}(z) \\
\rho_{k+1}(z) & \sigma_{k+1}(z)
\end{array}\right) .
$$

We have that

$$
\operatorname{det} C(0)=\operatorname{det}\left(\begin{array}{ll}
\rho_{0}(z) & \sigma_{0}(z) \\
\rho_{1}(z) & \sigma_{1}(z)
\end{array}\right)=1-z
$$

Since for $z=1$ (3.4) becomes of first order, it follows that $\operatorname{det} C(0) \neq 0$. This implies the thesis.

Theorem 3.6. Any solution of the difference equation (3.4) can be expressed as linear combination of $\rho_{k}(z)$ and $\sigma_{k}(z)$.

Proof. The proof is an easy consequence of the fact that $\rho_{k}(z)$ and $\sigma_{k}(z)$ are a basis for the space of solutions of (3.4).

Theorem 3.7. The polynomial $\hat{\rho}_{k-1}(z)$, defined in (2.15), satisfies the difference equation

$$
\hat{\rho}_{k}(z)=\frac{z+1}{2} \hat{\rho}_{k-1}(z)-\frac{(z-1)^{2}}{4\left(4-k^{-2}\right)} \hat{\rho}_{k-2}(z), \quad k \geq 1
$$

with initial conditions

$$
\hat{\rho}_{-1}(z)=0, \quad \hat{\rho}_{0}(z)=1 .
$$

Proof. From Theorem 3.4 and relation (2.15) the proof immediately follows.

We are now ready to answer the question concerning the values assumed by the polynomials $\hat{\rho}_{k-1}(z)$ and $\sigma_{k}(z)$ on the unit circle.

Theorem 3.8. Let $\sigma_{k}(z)$ be the polynomial satisfying the difference equation (3.4).

Then, for $m \geq 1$

$$
\sigma_{k}\left(e^{i \theta}\right)= \begin{cases}e^{i m \theta} f_{m}(\theta), & \text { if } k=2 m \\ \left(e^{i \theta}+1\right) e^{i m \theta} g_{m}(\theta), & \text { if } k=2 m+1\end{cases}
$$

with $f_{m}, g_{m}:[0,2 \pi) \rightarrow(0,+\infty)$.

Moreover, let $\hat{\rho}_{k-1}(z)$, be the polynomial satisfying the difference equation (3.17).

Then, for $m \geq 1$

$$
\hat{\rho}_{k-1}\left(e^{i \theta}\right)= \begin{cases}\left(e^{i \theta}+1\right) e^{i(m-1) \theta} \hat{f}_{m}(\theta), & \text { if } k=2 m \\ e^{i m \theta} \hat{g}_{m}(\theta), & \text { if } k=2 m+1\end{cases}
$$

with $\hat{f}_{m}, \widehat{g}_{m}:[0,2 \pi) \rightarrow(0,+\infty)$. 
Proof. We consider only the case related to the polynomial $\sigma_{k}(z)$, being the other one very similar.

The proof is by induction with respect to $m$. For $m=1$, from Theorem 3.2 it follows that

$$
\begin{aligned}
\sigma_{2}\left(e^{i \theta}\right) & =\frac{e^{i \theta}+1}{2} \sigma_{1}\left(e^{i \theta}\right)-\frac{\left(e^{i \theta}-1\right)^{2}}{12} \sigma_{0}\left(e^{i \theta}\right) \\
& =\left(\frac{e^{i \theta}+1}{2}\right)^{2}-\frac{\left(e^{i \theta}-1\right)^{2}}{12} \\
& =e^{i \theta} \frac{3\left(e^{i(\theta / 2)}+e^{-i(\theta / 2)}\right)^{2}-\left(e^{i(\theta / 2)}-e^{-i(\theta / 2)}\right)^{2}}{12} \\
& =e^{i \theta} \frac{2(\cos (\theta / 2))^{2}+1}{3}=e^{i \theta} \frac{(2+\cos \theta)}{3}=e^{i \theta} f_{1}(\theta) .
\end{aligned}
$$

Obviously, for each fixed $\theta \in[0,2 \pi), f_{1}(\theta)$ is a positive real number. Moreover,

$$
\begin{aligned}
\sigma_{3}\left(e^{i \theta}\right) & =\frac{e^{i \theta}+1}{2} \sigma_{2}\left(e^{i \theta}\right)-\frac{\left(e^{i \theta}-1\right)^{2}}{15} \sigma_{1}\left(e^{i \theta}\right) \\
& =\frac{e^{i \theta}+1}{2} e^{i \theta} \frac{(2+\cos \theta)}{3}-\frac{\left(e^{i \theta}-1\right)^{2}}{15} \frac{e^{i \theta}+1}{2} \\
& =\left(e^{i \theta}+1\right) e^{i \theta} \frac{10+5 \cos \theta+4(\sin (\theta / 2))^{2}}{30} \\
& =\left(e^{i \theta}+1\right) e^{i \theta} \frac{(4+\cos \theta)}{10}=\left(e^{i \theta}+1\right) e^{i \theta} g_{1}(\theta),
\end{aligned}
$$

with $g_{1}(\theta)$ a positive real number for all $\theta \in[0,2 \pi)$. We now show that if (3.19) holds for $m$, it also holds for $m+1$.

We only consider the case of $k$ even, because the proof for $k$ odd proceeds similarly. By applying (3.4) we obtain

$$
\begin{aligned}
\sigma_{2 m+2}\left(e^{i \theta}\right) & =\frac{e^{i \theta}+1}{2} \sigma_{2 m+1}\left(e^{i \theta}\right)-\frac{\left(e^{i \theta}-1\right)^{2}}{4\left[4-(2 m+1)^{-2}\right]} \sigma_{2 m}\left(e^{i \theta}\right) \\
& =\frac{\left(e^{i \theta}+1\right)^{2}}{2} e^{i m \theta} g_{m}(\theta)-\frac{\left(e^{i \theta}-1\right)^{2}}{4\left[4-(2 m+1)^{-2}\right]} e^{i m \theta} f_{m}(\theta) \\
& =\frac{\left[e^{i(\theta / 2)}\left(e^{i(\theta / 2)}+e^{-i(\theta / 2)}\right)\right]^{2}}{2} e^{i m \theta} g_{m}(\theta)-\frac{\left[e^{i(\theta / 2)}\left(e^{i(\theta / 2)}-e^{-i(\theta / 2)}\right)\right]^{2}}{4\left[4-(2 m+1)^{-2}\right]} e^{i m \theta} f_{m}(\theta) \\
& =e^{i(m+1) \theta}\left[2\left(\cos \frac{\theta}{2}\right)^{2} g_{m}(\theta)+\frac{(\sin (\theta / 2))^{2}}{4-(2 m+1)^{-2}} f_{m}(\theta)\right]=e^{i(m+1) \theta} f_{m+1}(\theta),
\end{aligned}
$$

where, for each fixed $\theta \in[0,2 \pi), f_{m+1}(\theta)$ is a positive real number.

This completes the induction step. 
Corollary 3.9. Suppose that the polynomials $\rho_{k}(z)$ and $\sigma_{k}(z)$, defined in (2.11), admit roots on the unit circle. Then, all of them are real and simple.

Proof. Since $f_{m}(\theta), g_{m}(\theta), \hat{f}_{m}(\theta)$ and $\hat{g}_{m}(\theta)$ are all positive in (3.19), (3.20) for all $\theta \in$ $[0,2 \pi)$, the only roots on $|z|=1$ are those coming from the factors $\left(e^{i \theta}+1\right)$ and $\left(e^{i \theta}-\right.$ $1)$.

\section{The stability problem for the top order family}

The results obtained in the previous section permit to attack the question concerning the stability problem for the $k$-step methods in the Top Order family, which will be used as BVMs with $(\nu, k-v)$-boundary conditions, where $v$ is defined according to (2.18).

Remark 4.1. From the statement (3) in Lemma 2.4 we have that $\rho_{k}(z)$ is of type $(r, k-$ $2 r, r)$, with $r$ a nonnegative integer. Analogously, we can derive that $\sigma_{k}(z)$ is of type $(s, k-$ $2 s, s)$, with $s \in \mathbb{N}$.

Theorem 4.2. Let $\rho_{k}(z)$ and $\sigma_{k}(z)$ be the polynomials defined in (2.11) and $v \geq 1$ given by (2.18). Then,

(1) $\rho_{k}(z)$ and $\sigma_{k}(z)$ are both of type $(\nu-1,1, v-1)$, for $k=2 v-1$;

(2) $\rho_{k}(z)$ is of type $(\nu-1,2, v-1)$ and $\sigma_{k}(z)$ is of type $(\nu, 0, v)$, for $k=2 v$.

Proof. From Corollary 3.9 it follows that, for $k=2 v-1$, the polynomials $\rho_{k}(z)$ and $\sigma_{k}(z)$ have only one root of unit modulus. Moreover, when $k=2 \nu, \rho_{k}(z)$ has two roots of unit modulus and $\sigma_{k}(z)$ has not roots of unit modulus. Then, the assertions of the theorem follow from Remark 4.1.

We are now in the position to give the main results related to the stability problem.

Theorem 4.3. Let $k=2 v-1$. The $k$-step methods in the Top Order family are perfectly $A_{\nu, \nu-1}$-stable.

Proof. By considering the map defined in (2.22) and using Theorem 3.8 we obtain that

$$
q_{k}\left(e^{i \theta}\right)=\frac{\left(e^{i \theta}-1\right) e^{i(v-1) \theta} \hat{g}_{\nu-1}(\theta)}{\left(e^{i \theta}+1\right) e^{i(\nu-1) \theta} g_{\nu-1}(\theta)},
$$

where $\hat{g}_{\nu-1}(\theta) / g_{\nu-1}(\theta)=G_{\nu-1}(\theta)$ is a positive real number for all $\theta \in[0,2 \pi)$. Consequently,

$q_{k}\left(e^{i \theta}\right)=\frac{\left(e^{i(\theta / 2)}-e^{-i(\theta / 2)}\right)}{\left(e^{i(\theta / 2)}+e^{-i(\theta / 2)}\right)} G_{\nu-1}(\theta)=\frac{2 i \sin (\theta / 2)}{2 \cos (\theta / 2)} G_{\nu-1}(\theta)=i \tan \frac{\theta}{2} G_{\nu-1}(\theta), \quad \forall \theta \in[0,2 \pi)$,

which is 0 for $\theta=0$ and $\infty$ for $\theta=\pi$. This implies that the boundary locus associated to the method coincides with the imaginary axis. It is understood that the north pole of the Riemann sphere in the $q$-plane is considered, because the boundary locus is unbounded. Consequently, it is a regular Jordan curve. According to the usual convention in complex analysis, we can conclude that the region of the $q$-plane internal to the boundary locus is the negative half complex plane. This completes the proof. 
It is worth to note that the $k$-step methods in the Top Order family having $k$ odd are known in literature as top order methods (TOMs) [2].

Theorem 4.4. Let $k=2 \nu$. The $k$-step methods in the Top Order family are $A_{\gamma, v}$-stable.

Proof. From (2.22) and Theorem 3.8 we obtain

$$
q_{k}\left(e^{i \theta}\right)=\frac{\left(e^{i 2 \theta}-1\right) e^{i(\nu-1) \theta} \hat{f}_{\nu}(\theta)}{e^{i \nu \theta} f_{\nu}(\theta)},
$$

where $2\left(\hat{f}_{\nu}(\theta) / f_{v}(\theta)\right)=F_{\nu}(\theta)$ is a positive real number for all $\theta \in[0,2 \pi)$. Consequently,

$$
q_{k}\left(e^{i \theta}\right)=\frac{\left(e^{i 2 \theta}-1\right)}{e^{i \theta}} \frac{F_{\nu}(\theta)}{2}=i \sin \theta F_{\nu}(\theta), \quad \forall \theta \in[0,2 \pi) .
$$

This implies that the boundary locus associated to the method coincides with the segment of the imaginary axis

$$
[-i F, i F], \quad \text { where } F=\max _{\theta \in[0,2 \pi)} F_{\nu}(\theta)
$$

Then, the region of $(\nu, \nu)$-Absolute stability is the whole complex plane excluding this segment. From Definition 2.2 the thesis follows.

\section{Conclusions}

In this paper it has been proved that the polynomials associated to the methods in the Top Order family satisfy linear difference equations with variable coefficients. A complete proof for the stability problem of such methods has been provided by using properties of the polynomials derived from these equations.

\section{References}

[1] L. Aceto, The Pascal matrix and some numerical methods for ODEs, Tech. Rep. 01/01, Dipartimento di Energetica, Università degli Studi di Firenze, Florence.

[2] L. Brugnano and D. Trigiante, Solving Differential Problems by Multistep Initial and Boundary Value Methods, Stability and Control: Theory, Methods and Applications, vol. 6, Gordon and Breach, Amsterdam, 1998.

[3] G. Dahlquist, Convergence and stability in the numerical integration of ordinary differential equations, Mathematica Scandinavica 4 (1956), 33-53.

[4] A. Gluchoff and F. Hartmann, Univalent polynomials and non-negative trigonometric sums, The American Mathematical Monthly 105 (1998), no. 6, 508-522.

[5] P. Henrici, Applied and Computational Complex Analysis, Pure and Applied Mathematics, vol. 1, John Wiley \& Sons, New York, 1974.

[6] J. J. H. Miller, On the location of zeros of certain classes of polynomials with applications to numerical analysis, Journal of the Institute of Mathematics and Its Applications 8 (1971), 397-406. 
14 Difference equations and the stability problem

[7] D. S. Mitrinović, Analytic Inequalities, Die Grundlehren der mathematischen Wisenschaften, vol. 1965, Springer, New York, 1970.

L. Aceto: Dipartimento di Matematica Applicata “U. Dini," Università di Pisa, Via Diotisalvi 2, 56126 Pisa, Italy

E-mail address: 1.aceto@dma.unipi.it

R. Pandolfi: Dipartimento di Matematica “U. Dini,” Università di Firenze, Viale Morgagni 67/A, 50134 Firenze, Italy

E-mail address: pandolfi@math.unifi.it

D. Trigiante: Dipartimento di Energetica “S. Stecco," Università di Firenze, Via C. Lombroso 6/17, 50134 Firenze, Italy

E-mail address: trigiant@unifi.it 